\title{
Hypernuclei in the Skyrme-Hartree-Fock formalism with a microscopic hyperon-nucleon force
}

\author{
J. Cugnon and A. Lejeune \\ Institut de Physique B5, Université de Liège, B-4000 Liège 1, Belgium \\ H.-J. Schulze \\ Departament d'Estructura i Constituents de la Matèria, Universitat de Barcelona, Av. Diagonal 647, E-08028 Barcelona, Spain
}

(Received 15 March 2000; published 13 November 2000)

\begin{abstract}
We determine properties of single and multilambda hypernuclei in the Skyrme-Hartree-Fock formalism, which is supplemented by a microscopic in-medium lambda-nucleon interaction derived from self-consistent Brueckner-Hartree-Fock calculations with the Nijmegen soft-core hyperon-nucleon potential. Existing data for single-lambda hypernuclei are well reproduced, apart from a slight underbinding of heavy nuclei. In multilambda hypernuclei, we study in particular the effects of the modification of the nuclear core due to the presence of the hyperons.
\end{abstract}

PACS number(s): 21.80.+a, 21.10.Dr, 21.30.Fe

\section{INTRODUCTION}

The experimental study of hypernuclei [1] is one of the few possibilities to constrain theoretical models of the bare hyperon-nucleon and hyperon-hyperon forces. Quantitative information on these forces is very important in particular for astrophysical applications [2].

However, it is then necessary to establish a link between the bare hyperon-nucleon force determined from scattering data and the effective in-medium force that is active in a nucleus. While many theoretical studies of hypernuclei have been performed that are based on phenomenological models (relativistic mean field [3,4], Skyrme-type [5], Woods-Saxon [6]) of the effective hyperon-nucleon interaction, we will test in this paper the capability of an entirely microscopical lambda hyperon-nucleon force, derived from BruecknerHartree-Fock (BHF) calculations of nuclear matter [7,8] with the Nijmegen soft-core hyperon-nucleon potential [9] (and the Paris nucleon-nucleon interaction), including explicitly the coupling of the lambda-nucleon to the sigma-nucleon states. It involves no adjustable parameters. There have been previous works $[10,11]$ on the properties of $\Lambda$-hypernuclei using a $\Lambda$-nucleon $G$-matrix that incorporates the short-range correlations. Also recently a Fermi hypernetted chain calculation was performed [12]. Our approach is somewhat more general, as we account for the effect of the hyperons on the nucleonic system, both in the description of the nucleus and, as we explain below, in the building of the effective $\Lambda$-nucleon interaction itself. This effect is not large in an ordinary hypernucleus with one hyperon. However, it is expected to be enhanced in many-lambda hypernuclei, of which we give an illustrative study.

The description of hypernuclei will be performed within the Skyrme-Hartree-Fock (SHF) formalism [13], where the microscopically derived effective $\Lambda$-nucleon interaction will be implemented. The nucleonic aspects of a hypernucleus being not the main focus of this paper, we will employ a phenomenological Skyrme force for the nucleon-nucleon interaction.

To ease the reading of this paper, let us mention readily some restrictions of our approach. First, in our BHF calcula- tions we neglect hyperon-hyperon interactions, because presently there is no information on scattering phase shifts, and consequently no reliable potentials are available. While this is not a problem for single- $\Lambda$ hypernuclei, the predictions for multilambda hypernuclei can only be qualitative, until good hyperon-hyperon interactions become available. Second, the effective lambda-nucleon force that we use here, is derived from BHF calculations of isospin symmetric nuclear matter and we consequently only consider nuclei that are nearly symmetric. A study of the isospin effects is deferred to future work. Third, our BHF calculations neglect three-baryon forces. From purely nucleonic calculations [14], it is known that up to normal nuclear matter density the contribution of such forces to the single-particle potentials is quite small (of the order of $1 \mathrm{MeV}$ ). Effects of similar size are expected for the $\Lambda$ single-particle potential due to $Y N N$ forces within a Brueckner approach, although different interactions arise from the virtual excitation of the lambda particle. Finally, as we explain below, our formalism does not allow to recuperate effective spin-orbit forces from the BHF results. However, experimentally, these forces appear small enough to be neglected at the present level of investigation [15].

The paper is divided as follows. In Sec. II, we present the formalism for deriving an effective interaction from microscopical BHF calculations. In Sec. III, we present results for observed hypernuclei with one or two lambda particles. We show that our approach provides results almost as good as phenomenological approaches. We also pay attention to the distortion of the nucleonic core by the hyperons and present results for multi- $\Lambda$ hypernuclei, for which these polarization effects are enhanced. Finally, a discussion of the results and our conclusions are contained in Sec. IV.

\section{FORMALISM}

Our model is based on the SHF model of nuclei developed and described in great detail in Ref. [13]. We extend this method to the description of hypernuclei by adding to the energy density functional a contribution due to the action of hyperon-nucleon forces. More precisely, the total energy of a nucleus in the extended SHF formalism is written as 


$$
E=\int d^{3} r \epsilon(r)
$$

with the energy density functional

$$
\epsilon=\epsilon_{N}\left[\tau_{n}, \tau_{p}, \rho_{n}, \rho_{p}, J_{n}, J_{p}\right]+\epsilon_{\Lambda}\left[\tau_{\Lambda}, \rho_{\Lambda}, \rho_{N}\right]
$$

and the local densities

$$
\begin{gathered}
\rho_{q}=\sum_{i=1}^{N_{q}}\left|\phi_{q}^{i}\right|^{2}, \\
\tau_{q}=\sum_{i=1}^{N_{q}}\left|\nabla \phi_{q}^{i}\right|^{2}, \\
\boldsymbol{J}_{q}=\sum_{i=1}^{N_{q}} \phi_{q}^{i *}\left(\nabla \phi_{q}^{i} \times \boldsymbol{\sigma}\right) / i,
\end{gathered}
$$

where $i$ denotes the occupied states, and $N_{q}$ is the number of particles of kind $q=n, p, \Lambda$.

We use the standard Skyrme functional for the purely nucleonic energy density, i.e., the term which would survive in the absence of hyperons,

$$
\begin{aligned}
\epsilon_{N}= & \frac{1}{2 m_{N}} \tau_{N}+\frac{t_{0}}{4}\left[\left(x_{0}+2\right) \rho_{N}^{2}-\left(2 x_{0}+1\right)\left(\rho_{n}^{2}+\rho_{p}^{2}\right)\right] \\
& +\frac{\left(t_{1}+t_{2}\right)}{4} \rho_{N} \tau_{N}+\frac{\left(t_{2}-t_{1}\right)}{8}\left(\rho_{n} \tau_{n}+\rho_{p} \tau_{p}\right) \\
& +\frac{\left(t_{2}-3 t_{1}\right)}{16} \rho_{N} \Delta \rho_{N}+\frac{\left(3 t_{1}+t_{2}\right)}{32}\left(\rho_{n} \Delta \rho_{n}+\rho_{p} \Delta \rho_{p}\right) \\
& +\frac{\left(t_{1}-t_{2}\right)}{16}\left(J_{n}^{2}+J_{p}^{2}\right)+\frac{t_{3}}{4} \rho_{n} \rho_{p} \rho_{N}+\frac{W}{2}\left(\rho_{N} \nabla \cdot J_{N}\right. \\
& \left.+\rho_{n} \nabla \cdot J_{n}+\rho_{p} \nabla \cdot J_{p}\right)+\epsilon_{\mathrm{Coul}},
\end{aligned}
$$

with $\rho_{N}=\rho_{n}+\rho_{p}$, etc. In the following we choose the parameters of the force SIII of Ref. [13].

We propose to construct the part of the energy density functional due to the presence of hyperons, $\epsilon_{\Lambda}$, as

$$
\epsilon_{\Lambda}=\frac{1}{2 m_{\Lambda}} \tau_{\Lambda}+\epsilon_{N \Lambda}\left(\rho_{N}, \rho_{\Lambda}\right)
$$

by requiring that it yields the additional binding energy per baryon, $B / A$, and the adequate $\Lambda$ effective mass, $m_{\Lambda}^{*}$, in uniform hypermatter, as generated by our previous BHF calculations, described in Ref. [8]. This means that, for uniform matter, the following relation must hold:

$$
\frac{1}{2 m_{\Lambda}} \tau_{\Lambda}+\epsilon_{N \Lambda}\left(\rho_{N}, \rho_{\Lambda}\right)=\left(\rho_{N}+\rho_{\Lambda}\right) \frac{B}{A}\left(\rho_{N}, \rho_{\Lambda}\right)-\rho_{N} \frac{B}{A}\left(\rho_{N}, 0\right) .
$$

In the absence of a lambda-lambda interaction one has

$$
\frac{1}{2 m_{\Lambda}} \tau_{\Lambda}=\rho_{\Lambda} \frac{B}{A}\left(0, \rho_{\Lambda}\right)=\frac{3}{5} \frac{\left(3 \pi^{2}\right)^{2 / 3}}{2 m_{\Lambda}} \rho_{\Lambda}^{5 / 3},
$$

where the last quantity is the binding energy per baryon of the ideal hyperon gas. One can thus write $\epsilon_{N \Lambda}$ as

$$
\begin{aligned}
\epsilon_{N \Lambda}\left(\rho_{N}, \rho_{\Lambda}\right)= & \left(\rho_{N}+\rho_{\Lambda}\right) \frac{B}{A}\left(\rho_{N}, \rho_{\Lambda}\right) \\
& -\rho_{N} \frac{B}{A}\left(\rho_{N}, 0\right)-\rho_{\Lambda} \frac{B}{A}\left(0, \rho_{\Lambda}\right) .
\end{aligned}
$$

The BHF binding energy per baryon of uniform hypermatter was determined in Ref. [8]. These calculations yield as functions of nucleonic and hyperonic partial densities $\rho_{N}$ and $\rho_{\Lambda},{ }^{1}$ the BHF single-particle potentials $U_{N}(k), U_{\Lambda}(k)$ of nucleons and hyperons, as well as the binding energy per baryon, $B / A$, that can in the absence of a lambda-lambda interaction be written as

$$
\begin{aligned}
\left(\rho_{N}+\rho_{\Lambda}\right) \frac{B}{A}= & 4 \sum_{k<k_{F}^{(N)}}\left[\frac{k^{2}}{2 m_{N}}+\frac{1}{2} U_{N}^{(N)}(k)\right] \\
& +2 \sum_{k<k_{F}^{(\Lambda)}}\left[\frac{k^{2}}{2 m_{\Lambda}}+U_{\Lambda}(k)\right] .
\end{aligned}
$$

The required energy density functional is therefore

$$
\begin{aligned}
\epsilon_{N \Lambda}\left(\rho_{N}, \rho_{\Lambda}\right)= & 2 \sum_{k<k_{F}^{(\Lambda)}} U_{\Lambda}(k) \\
& +2 \sum_{k<k_{F}^{(N)}}\left[\left.U_{N}^{(N)}(k)\right|_{\rho_{\Lambda}}-\left.U_{N}^{(N)}(k)\right|_{\rho_{\Lambda}=0}\right] .
\end{aligned}
$$

Here $U_{N}^{(N)}(k)$ is the part of the nucleon single-particle potential due to nucleons in the medium, see Ref. [8]. It depends only weakly (indirectly via the intermediate states of the Bethe-Goldstone equation) on the lambda density, so that the first term on the right-hand side (RHS) of the previous equation is the dominant one. In our model, the lambda singleparticle potential $U_{\Lambda}(k)$ determining that contribution is solely due to the interaction with the nucleons. A global $\Lambda$ effective mass can be defined by

$$
\frac{m_{\Lambda}^{*}}{m_{\Lambda}}=\left[1+\frac{U_{\Lambda}\left(k_{F}^{(\Lambda)}\right)-U_{\Lambda}(0)}{k_{F}^{(\Lambda) 2} / 2 m}\right]^{-1},
$$

such that

\footnotetext{
${ }^{1}$ The isospin-asymmetry of the nuclear matter is disregarded in this respect. It was shown in Ref. [2] that the effect of asymmetry on the hyperon single-particle potentials is not large. Also the nuclei we consider are nearly symmetric.
} 


$$
U_{\Lambda}(k) \approx U_{\Lambda}^{0}+\left(\frac{1}{2 m_{\Lambda}^{*}}-\frac{1}{2 m_{\Lambda}}\right) k^{2}+\cdots
$$

which actually approximates fairly well the true shape of the single-particle potential. Due to the fact that our BHF calculations neglect the hyperon-hyperon forces, the depth of the lambda single-particle potential as well as the lambda effective mass depend to a good approximation only on the nucleon partial density: $U_{\Lambda}^{0}\left(\rho_{N}\right), m_{\Lambda}^{*}\left(\rho_{N}\right)$. Also $U_{N}^{(N)}$ appearing in Eqs. (9) and (10) does not depend on $\rho_{\Lambda}$ in this approximation. Using Eqs. (10) and (12) one obtains in this case for the energy density due to hyperon-nucleon forces:

$$
\begin{aligned}
\epsilon_{N \Lambda}\left(\rho_{N}, \rho_{\Lambda}\right) \approx & 2 \sum_{k<k_{F}^{(\Lambda)}} U_{\Lambda}(k) \\
\approx & U_{\Lambda}^{0}\left(\rho_{N}\right) \rho_{\Lambda} \\
& +\left(\frac{m_{\Lambda}}{m_{\Lambda}^{*}\left(\rho_{N}\right)}-1\right) \frac{3}{5} \frac{\left(3 \pi^{2}\right)^{2 / 3}}{2 m_{\Lambda}} \rho_{\Lambda}^{5 / 3} .
\end{aligned}
$$

In the description of hypernuclei, we want to keep the motion of the hyperon as corresponding to an effective mass different from the bare mass, i.e., to the correct hyperon current. Therefore we rewrite Eq. (5) for $\epsilon_{\Lambda}$ as

$$
\epsilon_{\Lambda}=\frac{1}{2 m_{\Lambda}^{*}\left(\rho_{N}\right)} \tau_{\Lambda}+\epsilon_{N \Lambda}\left(\rho_{N}, \rho_{\Lambda}\right)+\Delta \epsilon\left(\rho_{N}, \rho_{\Lambda}\right) .
$$

The $\Delta \epsilon$ term arises from the substitution of $m_{\Lambda}$ by $m_{\Lambda}^{*}$ and should be such that the energy density recovers its BHF value for uniform matter. This requires that, in uniform matter,

$$
\frac{1}{2 m_{\Lambda}^{*}\left(\rho_{N}\right)} \tau_{\Lambda}+\Delta \epsilon\left(\rho_{N}, \rho_{\Lambda}\right)=\frac{1}{2 m_{\Lambda}} \tau_{\Lambda} .
$$

Using the relation between $\tau_{\Lambda}$ and $\rho_{\Lambda}$ as in Eq. (7), we finally get

$$
\begin{aligned}
\epsilon_{\Lambda}= & \frac{1}{2 m_{\Lambda}} \tau_{\Lambda}+\epsilon_{N \Lambda}\left(\rho_{N}, \rho_{\Lambda}\right) \\
& +\left(\frac{m_{\Lambda}}{m_{\Lambda}^{*}\left(\rho_{N}\right)}-1\right)\left(\frac{\tau_{\Lambda}}{2 m_{\Lambda}}-\frac{3}{5} \frac{\left(3 \pi^{2}\right)^{2 / 3}}{2 m_{\Lambda}} \rho_{\Lambda}^{5 / 3}\right)
\end{aligned}
$$

with $m_{\Lambda}^{*}$ being the lambda effective mass as determined in the BHF calculation.

Minimizing the total energy, one arrives with Eq. (16) at the SHF Schrödinger equation

$$
\begin{gathered}
{\left[-\boldsymbol{\nabla} \cdot \frac{1}{2 m_{q}^{*}(r)} \boldsymbol{\nabla}+V_{q}(r)-i W_{q}(r)(\boldsymbol{\nabla} \times \boldsymbol{\sigma})\right] \phi_{q}^{i}(r)} \\
\quad=-e_{q}^{i} \phi_{q}^{i}(r),
\end{gathered}
$$

with the single-particle energies $-e_{q}^{i}$ and the SHF mean fields

$$
\begin{aligned}
V_{N}= & V_{N}^{\mathrm{SHF}}+\frac{\partial \epsilon_{N \Lambda}}{\partial \rho_{N}} \\
& +\frac{\partial}{\partial \rho_{N}}\left(\frac{m_{\Lambda}}{m_{\Lambda}^{*}\left(\rho_{N}\right)}\right)\left(\frac{\tau_{\Lambda}}{2 m_{\Lambda}}-\frac{3}{5} \frac{\left(3 \pi^{2}\right)^{2 / 3}}{2 m_{\Lambda}} \rho_{\Lambda}^{5 / 3}\right), \\
V_{\Lambda}= & \frac{\partial \epsilon_{N \Lambda}}{\partial \rho_{\Lambda}}-\left(\frac{m_{\Lambda}}{m_{\Lambda}^{*}\left(\rho_{N}\right)}-1\right) \frac{\left(3 \pi^{2}\right)^{2 / 3}}{2 m_{\Lambda}} \rho_{\Lambda}^{2 / 3},
\end{aligned}
$$

where $V_{N}^{\text {SHF }}$ is the nucleonic Skyrme mean field without hyperons, as detailed in Ref. [13]. The nucleon mean field thus acquires a correction in the presence of hyperons, causing a rearrangement of the nucleonic structure of the hypernucleus. Note that in the case of an exact validity of Eq. (13), the SHF lambda mean field would correspond to the depth of the BHF single-particle potential: $V_{\Lambda}=U_{\Lambda}^{0}$. However, this relation is not exactly fulfilled, due to the various approximations that were made in order to arrive at Eq. (13).

There is no lambda spin-orbit mean field and the nucleon effective mass and spin-orbit mean field are not modified in our model. An approximate center of mass correction is applied as usual [13] by replacing the bare masses:

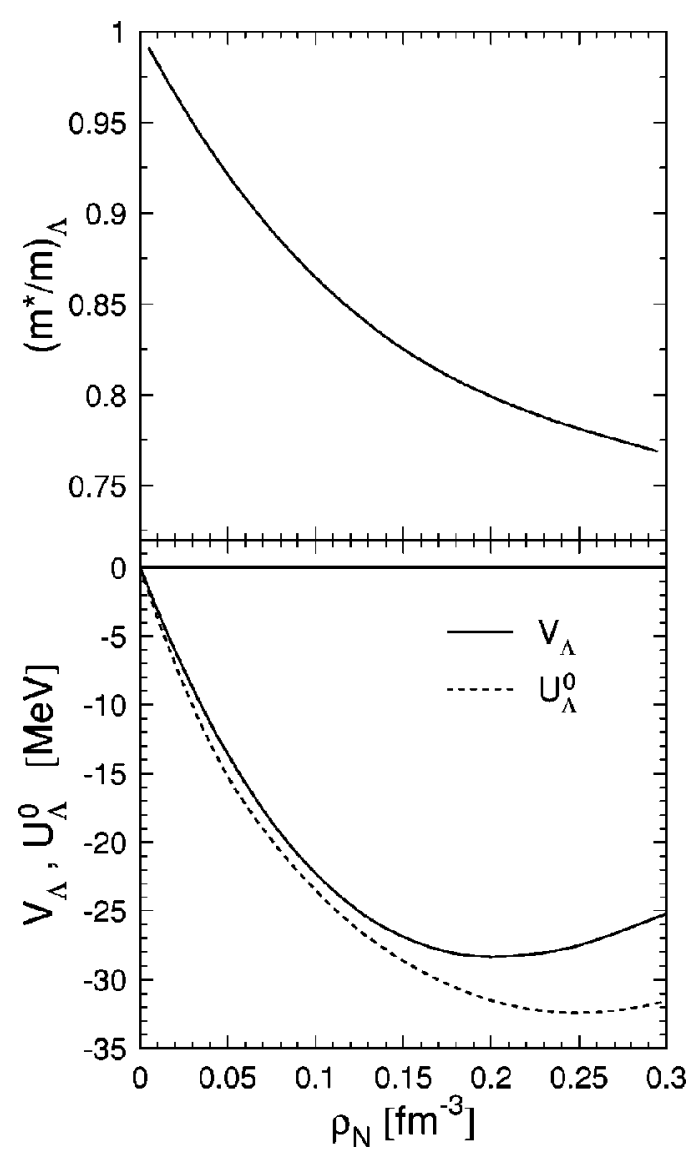

FIG. 1. Lambda effective mass (top panel) and well depth (bottom panel) as functions of nucleon density in pure nucleonic matter $\left(\rho_{\Lambda}=0\right)$. 


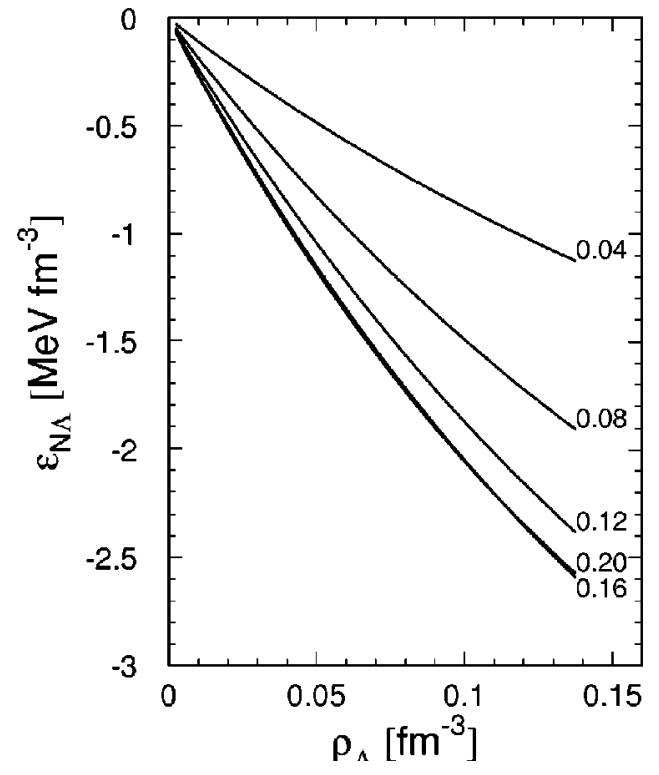

FIG. 2. Energy density of hypernuclear matter, Eq. (8), as a function of lambda density for different nucleon densities (indicated by the numbers near the curves).

$$
\frac{1}{m_{q}} \rightarrow \frac{1}{m_{q}}-\frac{1}{M}
$$

where $M=(N+Z) m_{N}+\Lambda m_{\Lambda}$ is the total mass of the nucleus.

In practice we use the following parametrizations of our numerical BHF results [8] for lambda effective mass and energy density $\left(\rho_{N}, \rho_{\Lambda}\right.$ given in $\mathrm{fm}^{-3}, \epsilon_{N \Lambda}$ in $\left.\mathrm{MeV} \mathrm{fm}^{-3}\right)$ :

$$
\begin{gathered}
\frac{m_{\Lambda}^{*}}{m_{\Lambda}}\left(\rho_{N}\right) \approx 1-1.83 \rho_{N}+5.33 \rho_{N}^{2}-6.07 \rho_{N}^{3}, \\
\epsilon_{N \Lambda}\left(\rho_{N}, \rho_{\Lambda}\right) \approx \\
+\left(327-1159 \rho_{N}+1163 \rho_{N}^{2}\right) \rho_{N} \rho_{\Lambda} \\
+\left(335-1102 \rho_{N}+1660 \rho_{N}^{2}\right) \rho_{N} \rho_{\Lambda}^{5 / 3},
\end{gathered}
$$

where the functional form of the parametrization Eq. (21) is suggested by Eq. (13). These quantities are displayed in Figs. 1 (top panel) and 2, respectively. At normal nuclear density, $\rho_{N}=\rho_{0}=0.17 \mathrm{fm}^{-1}$, we obtain in particular $m_{\Lambda}^{*} / m_{\Lambda} \approx 0.82$. Figure 1 shows also, for comparison with other approaches, the lambda mean field $V_{\Lambda}$, Eq. (18b), that appears in the Schrödinger equation, as well as the depth of the BHF single-particle potential $U_{\Lambda}^{0}=U_{\Lambda}(k=0)$, Eq. (12), as functions of nucleon density in pure nucleonic matter. As explained before, these two quantities are not identical. For $\rho_{\Lambda}=0$ one can derive the relation

$$
V_{\Lambda}=U_{\Lambda}^{0}+\left.2 \sum_{k<k_{F}^{(N)}} \frac{\partial U_{N}^{(N)}(k)}{\partial \rho_{\Lambda}}\right|_{\rho_{\Lambda}=0},
$$

where the second, "rearrangement," part on the RHS becomes more and more important with increasing nucleon

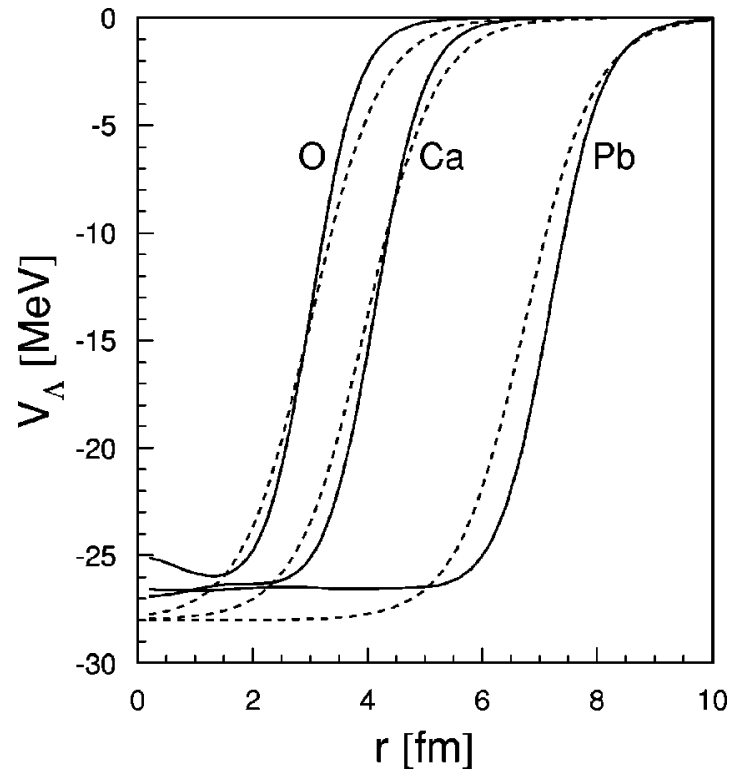

FIG. 3. SHF lambda mean field in ${ }_{\Lambda}^{17} \mathrm{O},{ }_{\Lambda}^{41} \mathrm{Ca}$, and ${ }_{\Lambda}^{209} \mathrm{~Pb}$ (solid lines). The dashed lines show the empirical Woods-Saxon parametrizations of Ref. [6].

density. Its value is about $+2 \mathrm{MeV}$ at saturation density and it increases nearly linearly with nucleon density. This can be observed in the lower panel of Fig. 1.

The value of the relevant mean field $V_{\Lambda}$ at saturation density is $V_{\Lambda}\left(\rho_{N}=\rho_{0}, \rho_{\Lambda}=0\right) \approx-28 \mathrm{MeV}$, and the maximum of binding is reached at a slightly higher density $\rho_{N}$ $\approx 0.20 \mathrm{fm}^{-3}$, comparable with, perhaps slightly less attractive than, typical phenomenological potentials of Refs. $[6,12]$. The high-density behavior cannot be directly constrained by hypernucleus data, however.

\section{RESULTS}

\section{A. Single and double-lambda hypernuclei}

As a first benchmark test of our method we performed calculations of single-lambda hypernuclei, where experimental information on single-particle levels is available.

We begin in Fig. 3 with a plot of the resulting SHF lambda mean field $V_{\Lambda}(r)$, Eq. (18b), for the hypernuclei ${ }_{\Lambda}^{17} \mathrm{O},{ }_{\Lambda}^{41} \mathrm{Ca}$, and ${ }_{\Lambda}^{209} \mathrm{~Pb}$. It is instructive to compare with the phenomenological lambda mean field that was derived in Ref. [6]. For the $\mathrm{Pb}$ nucleus, our theoretical potential extends slightly further than the phenomenological potential of that reference. However, ours has to be used in a wave equation with an effective mass. Making the usual coordinate transformation to eliminate the effective mass [16] would reduce the range of the resulting potential by a factor of the order of $\sqrt{m_{\Lambda}^{*} / m_{\Lambda}}$, i.e., by about $10 \%$. Our potential, so transformed, is similar to the one of Ref. [6], but a little bit less attractive, by about 1-2 MeV. In Skyrme-like approaches, the effective mass mocks up some finite range effects [13]. Other finite range effects can come from the folding of the $G$ matrix with the nuclear density matrix, a step beyond the local density approximation. This may have important consequences [12], although it is not clear whether the two effects are com- 
TABLE I. Lambda single-particle levels (in MeV) for different hypernuclei. The values in brackets are experimental data from Ref. [18] with errors of about $\pm 1 \mathrm{MeV}$. Also listed are rearrangement energies defined in Eqs. (23) and (24), as well as the ratios of hyperonic and nucleonic rms radii.

\begin{tabular}{lccccccccccccccc}
\hline \hline & ${ }_{\Lambda}^{13} \mathrm{C}$ & $\left({ }_{\Lambda}^{13} \mathrm{C}\right)$ & ${ }_{\Lambda}^{17} \mathrm{O}$ & $\left({ }_{\Lambda}^{16} \mathrm{O}\right)$ & ${ }_{\Lambda}^{29} \mathrm{Si}$ & $\left({ }_{\Lambda}^{28} \mathrm{Si}\right)$ & ${ }_{\Lambda}^{41} \mathrm{Ca}$ & $\left.{ }_{\Lambda}^{40} \mathrm{Ca}\right)$ & ${ }_{\Lambda}^{91} \mathrm{Zr}$ & $\left({ }_{\Lambda}^{89} \mathrm{Y}\right)$ & ${ }_{\Lambda}^{141} \mathrm{Ce}$ & $\left({ }_{\Lambda}^{139} \mathrm{La}\right)$ & ${ }_{\Lambda}^{209} \mathrm{~Pb}$ & $\left({ }_{\Lambda}^{208} \mathrm{~Pb}\right)$ \\
\hline $1 s$ & 11.7 & $(11.7)$ & 13.3 & $(12.5)$ & 16.4 & $(17.5)$ & 18.0 & $(20.0)$ & 21.1 & $(22.5)$ & 22.1 & $(24.0)$ & 23.1 & $(27.0)$ \\
$1 p$ & 0.9 & $(0.7)$ & 3.0 & $(2.5)$ & 7.4 & $(7.5)$ & 10.1 & $(12.0)$ & 15.6 & $(16.0)$ & 17.9 & $(21.0)$ & 19.6 & $(22.0)$ \\
$1 d$ & & & & & & & 1.6 & $(1.0)$ & 9.1 & $(9.0)$ & 12.8 & $(14.0)$ & 15.4 & $(17.0)$ \\
$1 f$ & & & & & & & & & 2.1 & $(2.0)$ & 6.9 & $(7.0)$ & 10.5 & $(12.0)$ \\
$1 g$ & & & & & & & & & & & 0.6 & $(1.0)$ & 5.1 & $(7.0)$ \\
$E_{R}$ & 1.22 & & 0.79 & & 0.52 & & 0.32 & & 0.11 & & 0.07 & & 0.02 & \\
$E_{R}^{\text {c.m. }}$ & 1.43 & 1.01 & & 0.59 & & 0.46 & & 0.23 & & 0.15 & & 0.11 \\
$R_{\Lambda} / R_{N}$ & 0.92 & & 0.89 & & 0.85 & & 0.81 & & 0.78 & & 0.78 & & & 0.75 \\
\hline \hline
\end{tabular}

pletely different from each other. This important issue should be addressed in future works following the folding procedure of Ref. [12].

The small lack of binding becomes visible in the comparison with experimental data: Table I and Fig. 4 show the lambda single-particle energies $e_{\Lambda}^{i}, i=1 s, 1 p, 1 d, 1 f$, for several hypernuclei. We observe a reasonable agreement with experimental results $[17,18]$, the theoretical predictions being, however, systematically slightly too small for heavy nuclei. A similar underbinding was also observed in the SHF approach of Ref. [11] with different hyperon-nucleon potentials. It may be partly related to the increasing isospin asymmetry of the heavier nuclei, that is not yet accounted for in the present model. The Nijmegen soft-core potential predicts more binding of the lambda in neutron-rich matter [2]. However, this will probably not be sufficient to compensate the observed lack of binding, which is therefore due to shortcomings of either the bare hyperon-nucleon potentials or the theoretical modeling of the hypernucleus (involving BHF and SHF stages of calculation). A first step to proceed will

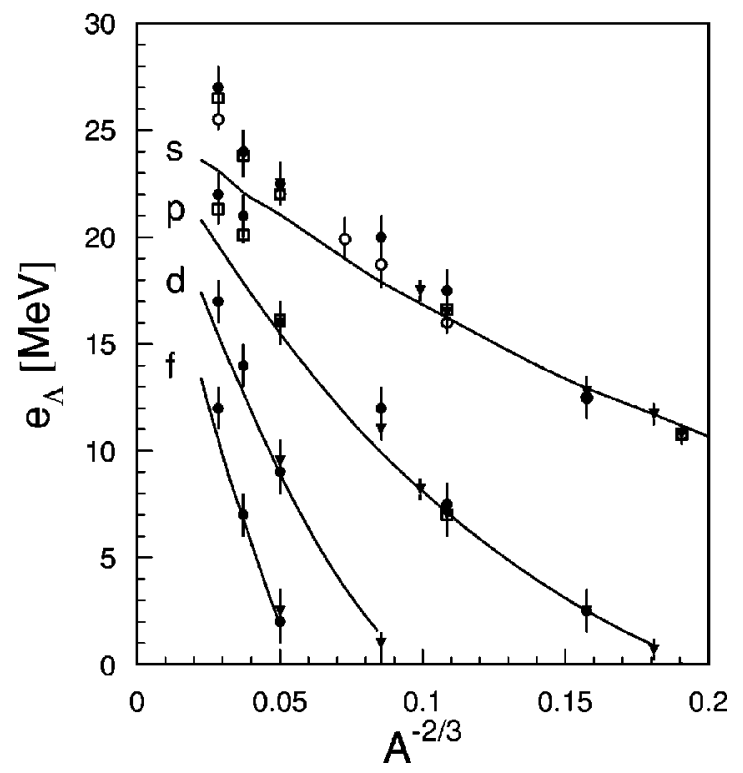

FIG. 4. Lambda single-particle energies for different hypernuclei as a function of mass number $A$ (solid lines). The markers indicate experimental data from Refs. [17,18]. be the application of our model with other hyperon-nucleon potentials in a future work.

Our approach enables us to determine the rearrangement energy (for the $1 s$ state)

$$
E_{R}=e_{1 s}^{\Lambda}+\left[E\left({ }_{\Lambda}^{A} Z\right)-E\left({ }^{A-1} Z\right)\right],
$$

that quantifies the contribution to the total energy coming from the change of binding of the nucleonic core caused by the presence of the $\Lambda$. We find generally $E_{R}>0$, i.e., the hypernucleus is less bound than indicated by the lambda single-particle energy. This means that the binding of the nucleonic component of the nucleus is reduced by the presence of the lambda. This change of binding has two distinct origins: One is the change of the nucleonic wave functions (core distortion) through the additional, $\rho_{\Lambda}$-dependent, term in Eq. (18a). This must increase the binding of the system $[5,11,19]$. However, as already stated in Ref. [5], we find that the dominant effect is coming from the different center of mass corrections, Eq. (19), that are applied to the nucleus with or without lambda. The rearrangement energy solely due to this procedure is positive and given by

$$
E_{R}^{\text {c.m. }}=\left(\frac{1}{M}-\frac{1}{M+m_{\Lambda}}\right) m_{N} E_{\mathrm{kin}} \approx \frac{1}{A^{2}} \frac{m_{\Lambda}}{m_{N}} E_{\mathrm{kin}},
$$

where $E_{\text {kin }}$ is the total kinetic energy of the nucleons in the nucleus. This value is listed for comparison in Table I. One sees that the effect can reach a magnitude of more than 1 $\mathrm{MeV}$ for very light nuclei, although the SHF approach is probably less reliable under these conditions. Inaccuracies due to the approximate treatment of c.m. corrections are consequently of the same order [5]. Nevertheless it demonstrates the importance of properly taking into account the backaction of the lambda on the nucleonic core. To be a bit more explicit, core distortion mainly originates from the second term on the RHS of Eq. (18a), the third one vanishing exactly in uniform matter. With the help of Eqs. (13) and (18a), one has in the limit of small lambda density:

$$
\frac{\partial \epsilon_{N \Lambda}}{\partial \rho_{N}} \approx \rho_{\Lambda} \frac{\partial U_{\Lambda}^{0}}{\partial \rho_{N}}
$$


TABLE II. Single-particle energy differences (in MeV) between lambda and neutron-hole states for different hypernuclei.

\begin{tabular}{ccccc}
\hline \hline Nucleus & $\Lambda$ & $n$ hole & Theor. & Expt. \\
\hline${ }^{12} \mathrm{C}$ & $1 s_{1 / 2}$ & $1 p_{3 / 2}$ & 5.6 & 6.7 \\
& $1 p_{3 / 2}$ & $1 p_{3 / 2}$ & 16.4 & 18.5 \\
& & & & \\
${ }^{16} \mathrm{O}$ & $1 p_{3 / 2}$ & $1 p_{3 / 2}$ & 18.1 & 19.2 \\
& $1 p_{1 / 2}$ & $1 p_{1 / 2}$ & 12.0 & 13.2 \\
& $1 s_{1 / 2}$ & $1 p_{3 / 2}$ & 7.6 & 9.9 \\
& $1 s_{1 / 2}$ & $1 p_{1 / 2}$ & 2.0 & 3.3 \\
& & & & \\
& & $1 d_{3 / 2}$ & 5.5 & 5.8 \\
${ }^{40} \mathrm{Ca}$ & $1 p_{1 / 2}$ & $1 d_{3 / 2}$ & 14.0 & 14.5 \\
& $1 d_{3 / 2}$ & $1 d_{5 / 2}$ & 20.5 & 19.4 \\
\hline \hline
\end{tabular}

Thus the crucial quantity, in this respect, is the derivative of $U_{\Lambda}^{0}$ with nucleon density. This point was already emphasized in Ref. [19].

Finally we list in the same Table I the ratios of hyperonic and nucleonic radii for the single-lambda hypernuclei: this quantity smoothly decreases with increasing mass number, mainly reflecting the fact that the lambda is lying more and more deeply in its potential well.

As another, simultaneous, test of hyperonic and nucleonic single-particle levels, we compare in Table II the experimental results for lambda-particle-neutron-hole excitation energies with the theoretical ones (taken as the differences of the single-particle energies) in ${ }_{\Lambda}^{12} \mathrm{C},{ }_{\Lambda}^{16} \mathrm{O}$, and ${ }_{\Lambda}^{40} \mathrm{Ca}$. For the nuclei considered, the agreement is reasonably good. This is in keeping with the remark already expressed that the lambda spin-orbit mean field is rather weak, presumably introducing shifts of not more than about $1 \mathrm{MeV}$.

The issue of core polarization is also important for the theoretical treatment of double-lambda hypernuclei [20]. The so far experimentally observed species $\left({ }_{\Lambda \Lambda}^{6} \mathrm{He},{ }_{\Lambda \Lambda}^{10} \mathrm{Be}\right.$, and probably ${ }_{\Lambda \Lambda}^{13}$ B) [21] are very light and the quantity of principal interest arises from a cancellation of large numbers. It is the bond energy of the lambda-lambda pair,

$$
\Delta B_{\Lambda \Lambda}=2 E\left({ }_{\Lambda}^{A-1} Z\right)-E\left({ }_{\Lambda \Lambda}^{A} Z\right)-E\left({ }^{A-2} Z\right),
$$

that is usually identified with the additional binding of the lambda-lambda pair, compared to twice the binding of a single lambda particle [22]. However, even without direct lambda-lambda interaction, the bond energy can be nonzero, and our model, since it does not contain a lambda-lambda interaction, allows to estimate the importance of this effect.

Our results are presented in Table III. We find a small negative bond energy, meaning that the binding of the nucleus increases weaker than linearly when adding lambdas. The center-of-mass corrections are nearly canceling in this case, $\Delta B_{\Lambda \Lambda}^{\text {c.m. }} \approx\left(2 / A^{3}\right)\left(m_{\Lambda} / m_{N}\right)^{2} E_{\text {kin }}$, and only for very light nuclei provide a significant, positive, contribution, whereas the total result is mainly due to the repulsive part of the energy density functional $\epsilon_{N \Lambda}$ : The second, repulsive, term on the RHS of Eqs. (13) or (21) depends stronger than linearly on the lambda density $\left(\sim \rho_{\Lambda}^{5 / 3}\right)$, and yields therefore more than twice the repulsion when going from the singlelambda to the double-lambda hypernucleus, leading to a negative value for the bond energy. The physical origin of this repulsive term is the momentum dependence of the BHF lambda single-particle potential, see Eq. (12), which produces the same effect as a small repulsive lambda-lambda force.

This effect is evidently independent of the modification of the (nucleonic and hyperonic) wave functions (rearrangement of the nuclear core), but in fact it is accompanied by a slight contraction of the nucleonic rms core radius $R_{N}$, that is also listed in Table III. One can see that the nucleonic core is very resistant to perturbation, although the core contraction is probably underestimated by our model, since the Skyrme force SIII yields a too large nuclear incompressibility $(355 \mathrm{MeV})$. For single-lambda hypernuclei, the change of the nucleonic radius (not shown) is about half the value indicated for double-lambda hypernuclei. Concerning the bond energy itself, if the experimental values of about $+5 \mathrm{MeV}$ are confirmed, a rather attractive lambda-lambda force will be implied. Note, however, that since those double-lambda hypernuclei presumably show an $\alpha$ structure, a cluster approach is certainly needed to extract reliable information, as underlined in Refs. [22,23].

\section{B. Multilambda hypernuclei}

Although our model does not comprise hyperon-hyperon interactions, we will apply it in the following to the description of multilambda hypernuclei. Our goal is primarily to study the rearrangement of the nuclear core under the influence of many lambdas and, secondly, to establish lower bounds on certain quantities like the lambda drip line. Our investigation may appear as academic. However, multilambda hyperfragments are presumably produced already in high energy collisions and may perhaps be detected soon [24]. Furthermore, if the $\Lambda-\Lambda$ interaction is attractive, as alluded above, our calculation may provide upper bounds for certain quantities. Of course, the lifetimes of these systems are presumably quite short and the investigation of this topic is outside the scope of this paper.

TABLE III. Bond energies [Eq. (26)] and relative contraction of the nucleonic core $\left(R_{N}\right.$ is the nucleonic rms radius) of several double-lambda hypernuclei.

\begin{tabular}{lcccccccc}
\hline \hline & ${ }_{\Lambda \Lambda}^{10} \mathrm{Be}$ & ${ }_{\Lambda \Lambda}^{14} \mathrm{C}$ & ${ }_{\Lambda \Lambda}^{18} \mathrm{O}$ & ${ }_{\Lambda \Lambda}^{30} \mathrm{Si}$ & ${ }_{\Lambda \Lambda}{ }^{42} \mathrm{Ca}$ & ${ }_{\Lambda \Lambda} \mathrm{Zr}$ & ${ }_{\Lambda \Lambda}^{142} \mathrm{Ce}$ & ${ }_{\Lambda \Lambda}^{210} \mathrm{~Pb}$ \\
\hline$\Delta B_{\Lambda \Lambda}[\mathrm{MeV}]$ & -0.34 & -0.41 & -0.41 & -0.33 & -0.31 & -0.21 & -0.14 & -0.12 \\
$\delta R_{N} / R_{N}[\%]$ & -1.08 & -0.56 & -0.61 & -0.33 & -0.31 & -0.16 & -0.14 & -0.10 \\
\hline \hline
\end{tabular}




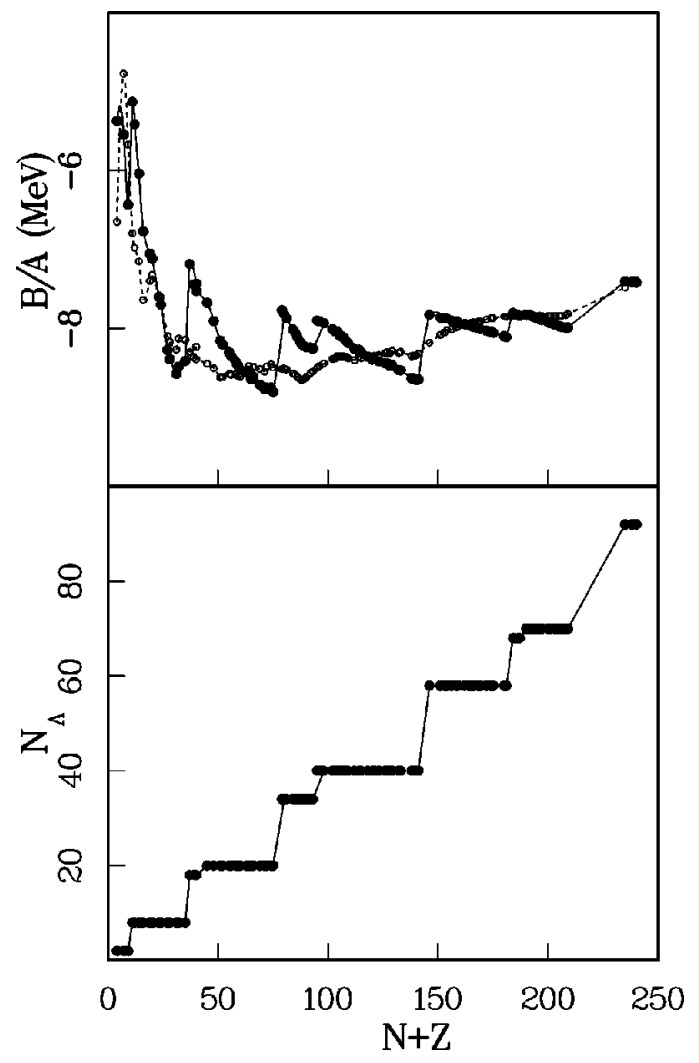

FIG. 5. Bottom panel: Lambda drip line. The heavy dots indicate the maximum number $N_{\Lambda}$ of lambda particles that can be attached to selected nuclei of mass number $N+Z$ along the stability line. Top panel: Binding energy per baryon for ordinary nuclei along the stability line (open dots) and for hypernuclei along the lambda drip line (full dots), plotted against the number of nucleons $N+Z$. The continuous lines are to guide the eye.

In the lower part of Fig. 5, we present the lambda drip line, i.e., the maximum number of $\Lambda$ 's that can be bound to a nucleus, corresponding to a vanishing $\Lambda$ chemical potential. This number is surprisingly large, but in agreement with the maximum hyperon content in uniform matter, as calculated in Refs. [7,8], i.e., about $1 / 3$ of the number of nucleons. It is however smaller than in some relativistic mean field calculations $[3,4]$, because usually in those works an attractive lambda-lambda interaction is assumed. We also disregard the possibility of populating the hypernucleus with cascade hyperons via the $\Lambda \Lambda \rightarrow N \Xi$ reaction [4], because it depends primarily on the binding of the $\Xi$ in nuclear matter, which is not known at the moment.

The drip line in Fig. 5 increases by steps because, when an additional lambda single-particle level becomes bound with increasing number of nucleons, it can readily be filled with $\Lambda$ particles without very much disturbing the effective force generated by the nucleons, and the maximum number can therefore only further increase by the binding of another level. This so occurs because of our neglect of hyperonhyperon interactions. In a more realistic calculation including $\Lambda-\Lambda$ interaction, the maximum number of lambdas would supposedly increase more regularly.

The upper part of Fig. 5 gives the binding energy per baryon of the nuclei along the $\Lambda$ drip line and compares it with the same quantity for stable nuclei (the usual BetheVon Weizsäcker plot). ${ }^{2}$ On the average, the binding energy per baryon is not changed sizably when going from the stability line to the drip line. Whenever a new $\Lambda$ single-particle level becomes just bound, new hyperons can be added on this level and the energy per baryon is suddenly reduced (in magnitude). Thus, just before a new single-particle level is bound, the energy per baryon is larger than the same quantity for the nucleon core.

The matter distribution is displayed in Fig. 6 for some of these hypernuclei, as well as the corresponding rms radii, in Fig. 7. One observes that the $\Lambda$ rms radius is increasing steadily with the number of hyperons, whereas the neutron and proton rms radii remain basically constant. In fact, although it is hardly visible in Fig. 7, they decrease slightly for small numbers of hyperons, consistently with the results for single and double- $\Lambda$ hypernuclei (see above). This result, also obtained in previous studies $[3,5,10]$, emphasizes the strong resistance of the nucleonic core to the perturbation brought by the added hyperons. The small irregularities in the curve of the $\Lambda \mathrm{rms}$ radius are due to the steplike filling of the hyperon orbitals, as we already explained. For a small number of $\Lambda$ particles, the latter are occupying low-lying orbitals with a small spatial extension. As a result, the $\Lambda \mathrm{rms}$ radius is smaller than the one of the nucleonic core. For a large number of $\Lambda$ particles, close to the drip line, the hyperons occupy all orbitals, up to barely bound ones, which have a large extension. This generates a large $\Lambda$ rms radius, overshooting the one of the nucleonic core.

One may summarize these considerations as follows. The nucleonic core provides an attractive potential for the $\Lambda$ particles, in which they can accumulate on the various singleparticle states without sizably disturbing the nucleonic core and each other. Let us notice, however, that the $\Lambda$ potential $V_{\Lambda}$ is progressively reduced when $\Lambda$ particles are added. At the drip line, its depth is about $20 \%$ smaller than for single$\Lambda$ hypernuclei.

As tiny as it can be, the distortion of the nucleonic core nevertheless presents interesting features as shown by the right panel of Fig. 6. The latter displays the change of the neutron density profile for different numbers of added $\Lambda$ particles (the proton density profiles exhibit basically the same features). When this number is low, the neutron density in the interior of the nucleus is slightly enhanced (core contraction, as observed for single and double hypernuclei). Of course, this is accompanied by a small depression at the nuclear surface, as the total neutron number is kept constant. The interior enhancement originates from the basically attractive nature of the lambda-nucleon interaction. When the number of $\Lambda$ particles is increasing, the repulsive part of the interaction is coming more and more into play (as the mean lambda-nucleon distance is diminishing), the nucleons are slightly repelled, and their density in the interior is depressed a little bit. This behavior is in keeping with the calculations

\footnotetext{
${ }^{2}$ Note that in this plot, the comparison is not done at fixed baryon number, but at fixed nucleon number.
} 


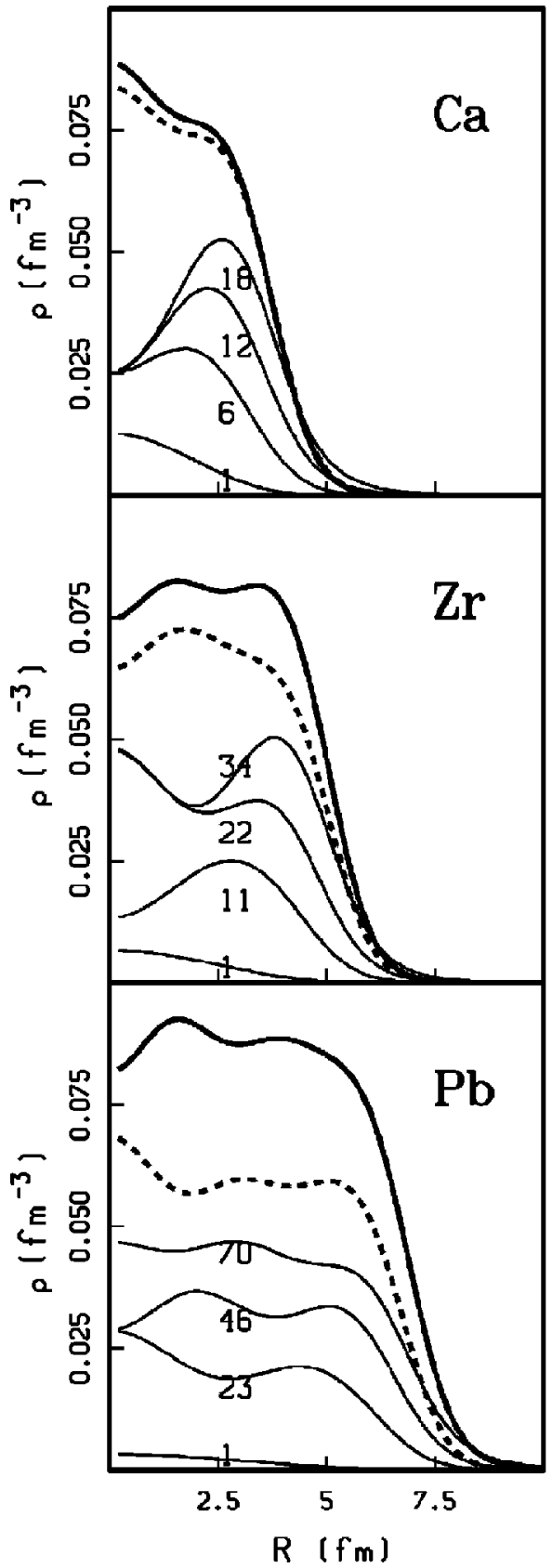

of uniform hypermatter, which show that the saturation baryon density stays approximately constant with increasing lambda fraction (see Fig. 5 of Ref. [8]), meaning that the nucleon density decreases. Outside the nuclear interior, the modification of the neutron density presents an oscillatory behavior. This is reminiscent of Friedel oscillations, although such an origin can hardly be assigned, in view of the nonuniformity of the unperturbed nucleon density in the region where these oscillations appear.

The $\Lambda$ density profiles reflect the progressive filling of the single-particle states. For small values of the $\Lambda$-particle number, the $\Lambda$ density falls off more rapidly than the nucleon density, whereas the situation is reversed for $\Lambda$-rich hypernuclei. This last situation is due to the occupation of barely bound orbits and bears some resemblance with neutron halo nuclei. Similarly, these $\Lambda$-rich hypernuclei should show ab-
FIG. 6. Density profiles of hypernuclei containing a ${ }^{40} \mathrm{Ca},{ }^{90} \mathrm{Zr}$, or ${ }^{208} \mathrm{~Pb}$ nucleonic core (from top to bottom in each panel). In the left panel, the heavy lines correspond to the neutron (full lines) and the proton (dotted lines) density profiles for the ordinary nuclei (no lambda particle). The thin lines give the lambda density profiles for hypernuclei with a number of $\Lambda$ particles (indicated near the curves) equal, respectively, to about $1 / 3,2 / 3$, and $3 / 3$ of the value at the drip line, as given in Fig. 5. The right panel displays the change in the neutron density profile, compared to the ordinary nuclei, when 1/3 (dotted lines), 2/3 (dashed lines), or 3/3 (full lines) of the drip line value of $\Lambda$ particles are added. In all plots, also the results for single-lambda hypernuclei are displayed for comparison. 


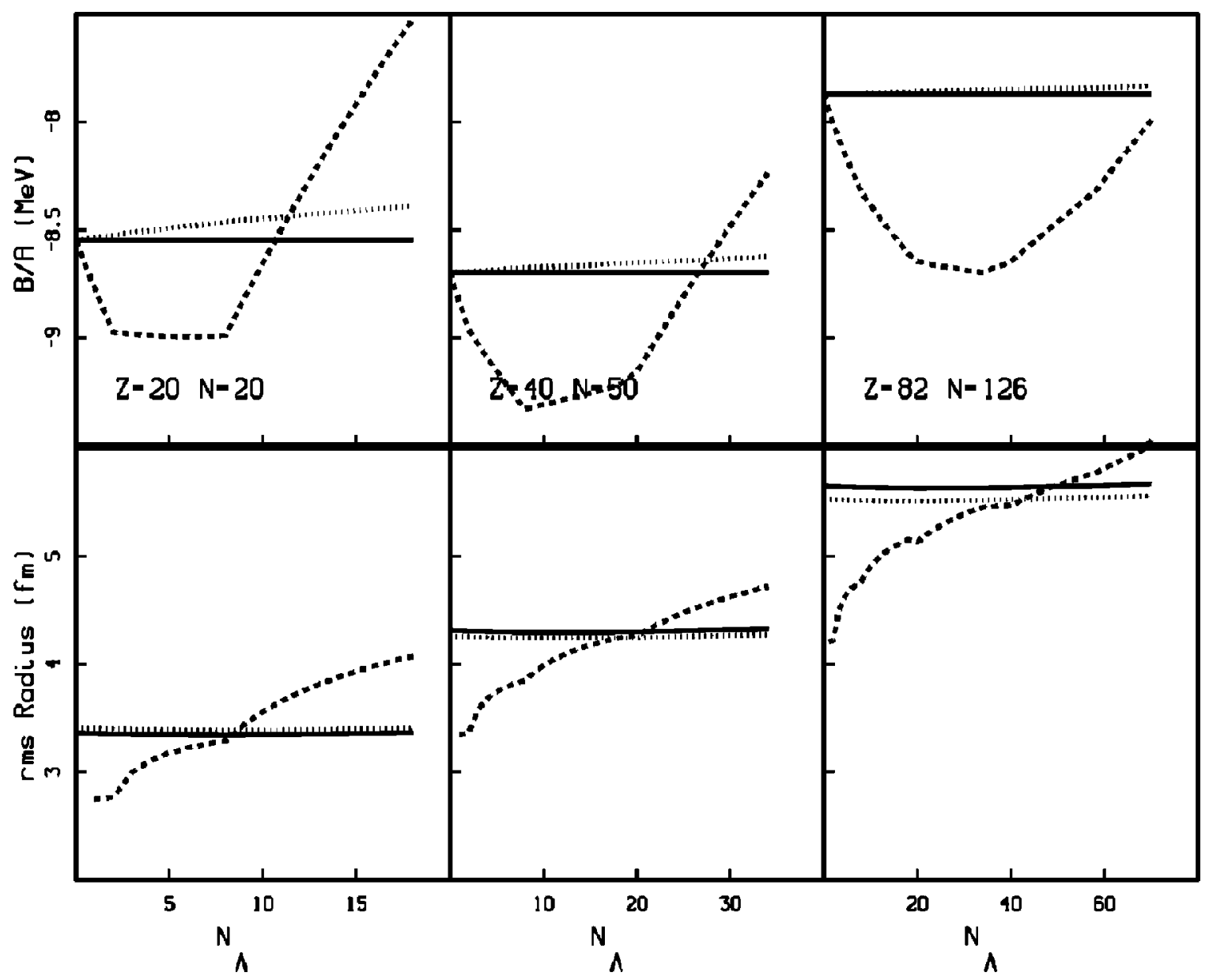

FIG. 7. Binding energy per baryon (top panel) and rms radii (bottom panel) for hypernuclei containing a ${ }^{40} \mathrm{Ca}$, ${ }^{90} \mathrm{Zr}$, or ${ }^{208} \mathrm{~Pb}$ nucleonic core (from left to right in each panel). In the top panel, the horizontal solid lines correspond to the binding energy per nucleon of the ordinary nuclei (no lambda particle). The dashed lines give the binding energy per baryon of hypernuclei with a varying number of $\Lambda$ particles $N_{\Lambda}$. The dotted lines give the nucleonic part of the binding energy per nucleon, i.e., the part coming from the quantity $\epsilon_{N}$ in Eqs. (1) and (2). The bottom panel displays the neutron (full lines), proton (dotted lines), and lambda (dashed lines) rms radii.

core. This has to cause a reduction in binding, since the configuration of the core without hyperons corresponds to the minimized energy of the purely nucleonic Hamiltonian. The effect, although weak, is clearly visible in Fig. 7 (dotted curves).

The maximum gain in binding energy is substantially larger than in uniform matter (see Ref. [7]). This is mainly due to the reduction of the nucleon binding energy (per nucleon) in finite nuclei, compared to nuclear matter. On the other hand, the $\Lambda$ potential has basically the same depth in finite nuclei and in uniform matter. Therefore, in hypernuclei with a moderate number of $\Lambda$ particles, the hyperons provide an additional binding energy per particle larger than in infinite matter.

\section{CONCLUSIONS}

In this paper, the SHF model was extended to the description of hypernuclei by supplementing its energy density functional with a contribution due to hyperon-nucleon forces, derived from self-consistent BHF calculations of hypernuclear matter with the Nijmegen soft-core potential. The lambda-lambda interaction was disregarded.

We have shown that our model is able, without any adjustable parameter, to give reliable results for single-lambda hypernuclei. These results are slightly less satisfactory than those obtained with phenomenological relativistic or nonrelativistic potentials, which are fitted, at least partially, to the hypernuclei data. The remaining discrepancies (underbinding for heavy nuclei) between theoretical and experimental results are possibly due to the quality of the bare forces, but perhaps also due to the theoretical modeling of hypernuclear matter (lowest order BHF without three-baryon forces, neglecting the isospin asymmetry) and hypernuclei. (The SHF approach neglects effects due to the finite range of the interactions and might not be adequate for light nuclei.) A first step towards clarifying these questions will be a comparison of results using other nucleon-hyperon potentials (in particular the new Nijmegen potentials [25] that also comprise hyperon-hyperon interactions) within the present approach. Further experimental efforts in the determination of scattering phase shifts and spectra of hypernuclei are however needed before a precise and complete determination of 
hyperon-nucleon (and in particular hyperon-hyperon) bare forces is feasible.

We want to comment on the consequences of neglecting three-body forces. Three-nucleon forces are expected to have very little effects on the $\Lambda$ single-particle potential. More important effects can arise from $\Lambda N N$ interactions coming from the intermediate hyperon excitation to a $\Sigma$ state, as underlined in Ref. [12]. In this reference a distinction is made between dispersive forces and two-pion forces (see Fig. 2 of the reference). Since the Nijmegen interaction that we used explicitly introduces the coupling between the $\Lambda N$ and the $\Sigma N$ channels and since nucleons are dressed in Brueckner $G$-matrix calculations, the dispersive force effects are included in our lowest-order Brueckner calculations. The two-pion $\Lambda N N$ forces are not included however. According to Ref. [12], their effects depend sensitively upon the $\Lambda \mathrm{N}$ correlations, but are basically attractive. On the other hand, our calculations neglect finite-range effects by adopting a local density approximation [Eq. (18)]. In Ref. [12], it is shown that these finite-range effects might give a strong repulsion, of the order of $2 \mathrm{MeV}$, in the single-particle binding energies in heavy nuclei. Our satisfactory results might then come from the compensation between these two neglected effects. This question would certainly deserve further detailed investigations.

We have also performed an exploratory study of multilambda hypernuclei. We found that the lambda drip line corresponds to a maximum $\Lambda$ content of about one third. If the hyperon-hyperon interaction, that we neglected, is attractive, this number can be considered as a lower limit. We also investigated the influence of the lambda particles on the nucleonic core, paying particular attention to the nonlinearities (in the densities) of the interaction. We found, as in previous mean field studies, that the nucleonic core is very resistant to the perturbation caused by the hyperons, even for a large number of hyperons. This result seems thus well established and is not expected to change drastically by the introduction of a lambda-lambda interaction.

\section{ACKNOWLEDGMENTS}

We thank Nguyen Van Giai and D. Vautherin for providing us with their SHF code and A. Polls and A. Ramos for helpful discussions. This work was supported in part by the program "Estancias de científicos y tecnólogos extranjeros en España."
[1] T. Motoba, H. Bandō, K. Ikeda, and T. Yamada, Prog. Theor. Phys. Suppl. 81, 42 (1985); H. Bandŏ, T. Motoba, and J. Žofka, Int. J. Mod. Phys. A 5, 4021 (1990); H. Feshbach, Nucl. Phys. A507, 219c (1990); B. F. Gibson and E. V. Hungerford III, Phys. Rep. 257, 349 (1995).

[2] M. Baldo, G. F. Burgio, and H.-J. Schulze, Phys. Rev. C 58, 3688 (1998); 61, 055801 (2000); I. Vidaña, A. Polls, A. Ramos, L. Engvik, and M. Hjorth-Jensen, ibid. 62, 035801 (2000).

[3] J. Mareš and J. Žofka, Z. Phys. A 333, 209 (1989); 345, 47 (1993); M. Rufa, J. Schaffner, J. Maruhn, H. Stöcker, W. Greiner, and P.-G. Reinhard, Phys. Rev. C 42, 2469 (1990); N. K. Glendenning, D. Von-Eiff, M. Haft, H. Lenske, and M. K. Weigel, ibid. 48, 889 (1993); J. Mareš and B. K. Jennings, ibid. 49, 2472 (1994); K. Tsushima, K. Saito, and A. W. Thomas, Phys. Lett. B 411, 9 (1997); D. Vretenar, W. Pöschl, G. A. Lalazissis, and P. Ring, Phys. Rev. C 57, R1060 (1998).

[4] J. Schaffner, C. Greiner, and H. Stöcker, Phys. Rev. C 46, 322 (1992); J. Schaffner, C. B. Dover, A. Gal, C. Greiner, D. J. Millener, and H. Stöcker, Ann. Phys. (N.Y.) 235, 35 (1994).

[5] M. Rayet, Ann. Phys. (N.Y.) 102, 226 (1976); Nucl. Phys. A367, 381 (1981).

[6] D. J. Millener, C. B. Dover, and A. Gal, Phys. Rev. C 38, 2700 (1988).

[7] H.-J. Schulze, M. Baldo, U. Lombardo, J. Cugnon, and A. Lejeune, Phys. Lett. B 355, 21 (1995).

[8] H.-J. Schulze, M. Baldo, U. Lombardo, J. Cugnon, and A. Lejeune, Phys. Rev. C 57, 704 (1998).

[9] P. Maessen, Th. Rijken, and J. de Swart, Phys. Rev. C 40, 2226 (1989).

[10] Y. Yamamoto, H. Bandō, and J. Žofka, Prog. Theor. Phys. 80,
757 (1988); Y. Yamamoto and H. Bandō, ibid. 83, 254 (1990); D. Halderson, Phys. Rev. C 48, 581 (1993); 61, 034001 (2000); J. Hao, T. T. S. Kuo, A. Reuber, K. Holinde, J. Speth, and D. J. Millener, Phys. Rev. Lett. 71, 1498 (1993); Y. Yamamoto, T. Motoba, H. Himeno, K. Ikeda, and S. Nagata, Prog. Theor. Phys. Suppl. 117, 361 (1994); I. Vidaña, A. Polls, A. Ramos, and M. Hjorth-Jensen, Nucl. Phys. A644, 201 (1998); Y. Tzeng, S. Y. T. Tzeng, T. T. S. Kuo, and T.-S. H. Lee, Phys. Rev. C 60, 044305 (1999).

[11] D. E. Lanskoy and Y. Yamamoto, Phys. Rev. C 55, 2330 (1997).

[12] Q. N. Usmani and A. R. Bodmer, Phys. Rev. C 60, 055215 (1999).

[13] D. Vautherin and D. M. Brink, Phys. Rev. C 5, 626 (1972); M. Beiner, H. Flocard, Nguyen Van Giai, and P. Quentin, Nucl. Phys. A238, 29 (1975).

[14] R. B. Wiringa, V. Fiks, and A. Fabrocini, Phys. Rev. C 38, 1010 (1988); A. Lejeune, U. Lombardo, and W. Zuo, Phys. Lett. B 477, 45 (2000).

[15] C. B. Dover et al., Phys. Rep. 184, 1 (1989).

[16] P. E. Hodgson, The Nucleon Optical Model (World Scientific, Singapore, 1994).

[17] B. Povh, Nucl. Phys. A335, 233 (1980); R. E. Chrien, ibid. A478, 705c (1988); P. H. Pile, Phys. Rev. Lett. 66, 2585 (1991); T. Hasegawa et al., Phys. Rev. C 53, 1210 (1996).

[18] S. Ajimura et al., Nucl. Phys. A585, 173c (1995).

[19] Q. N. Usmani and A. R. Bodmer, in Condensed Matter Theories, edited by J. W. Clark, K. A. Shoaib, and A. Sadig (Nova, Commack, NY, 1994), p. 395; A. R. Bodmer, S. Murali, and Q. N. Usmani, Nucl. Phys. A609, 326 (1996).

[20] K. Ikeda, H. Bandŏ, and T. Motoba, Prog. Theor. Phys. Suppl. 81, 147 (1985); S. B. Carr, I. R. Afnan, and B. F. Gibson, 
Nucl. Phys. A625, 143 (1997); S. Marcos, R. J. Lombard, and J. Mareš, Phys. Rev. C 57, 1178 (1998); D. E. Lanskoy, ibid. 58, 3351 (1998); K. Tominaga, T. Ueda, M. Yamaguchi, N. Kijima, D. Okamoto, K. Miyagawa, and T. Yamada, Nucl. Phys. A642, 483 (1998); J. Caro, C. García-Recio, and J. Nieves, ibid. A646, 299 (1999).

[21] M. Danysz et al., Nucl. Phys. 49, 121 (1963); J. Prowse, Phys. Rev. Lett. 17, 782 (1966); S. Aoki et al., Prog. Theor. Phys. 85, 1287 (1991); D. H. Davis, Nucl. Phys. A547, 369c (1992); G. B. Franklin, ibid. A585, 83c (1995).

[22] R. H. Dalitz, D. H. Davis, P. H. Fowler, A. Montwill, J.
Pniewski, and J. A. Zakrzewski, Proc. R. Soc. London, Ser. A 426, 1 (1989).

[23] A. R. Bodmer and Q. N. Usmani, Nucl. Phys. A468, 653 (1987).

[24] J. Žofka et al., Phys. Lett. B 235, 25 (1990); A. J. Baltz et al., ibid. 325, 7 (1994); P. H. Pile, in Properties and Interactions of Hyperons, edited by B. J. Gibson, P. D. Barnes, and K. Nakai (World Scientific, Singapore, 1994).

[25] Th. A. Rijken, V. G. J. Stoks, and Y. Yamamoto, Phys. Rev. C 59, 21 (1999); V. G. J. Stoks and Th. A. Rijken, ibid. 59, 3009 (1999). 